\title{
ORIGINAL ARTICLE ProHNPs are specific markers of normal myelopoiesis
}

\author{
F Emmertsen, A Glenthøj, J Sønderskov, P Kampmann, H Sengeløv and N Borregaard
}

Pro human neutrophil peptides (proHNP)s are proforms of $\alpha$-defensins produced by precursors of human neutrophils. They are secreted to bone marrow plasma in large amounts by myelocytes. We hypothesized that the plasma concentration of proHNPs might serve as a specific marker of myelopoietic activity, heralding the onset of normal myelopoiesis before reappearance of neutrophils, in the setting of bone marrow regeneration. To investigate this, plasma levels of proHNPs were measured by enzymelinked immunosorbent assay in blood samples collected from patients undergoing allogeneic $(n=11)$ or autologous $(n=16)$ stem cell transplantations (SCTs) and patients receiving chemotherapy for acute leukemia $(n=14)$. To compare proHNPs with previously suggested myeloid markers, myeloperoxidase (MPO), lysozyme and neutrophil gelatinase-associated lipocalin (NGAL) were also assayed. In all but one patient, chemotherapy led to the complete disappearance of ProHNPs from plasma. It reappeared in plasma on average 6.3 days before reappearance of neutrophils in the allogeneic setting, whereas this was reduced to an average of 2.8 days in the autologous SCT patients who received granulocyte colony-stimulating factor. Patients with acute myeloid leukemia $(n=19)$ had significantly lower levels of plasma proHNPs than healthy controls, indicating that proHNPs are not produced by leukemic blasts. We conclude that plasma concentration of proHNPs is a clinically useful marker of normal myelopoiesis.

Blood Cancer Journal (2014) 4, e193; doi:10.1038/bcj.2014.11; published online 21 March 2014

\section{INTRODUCTION}

Neutrophils are essential for our defense against microbial infection. Their short lifetime after release from the bone marrow necessitates an extensive production in the order of 0.8 to 1 billion neutrophils/kg bodyweight/day by the bone marrow. ${ }^{1}$ As the production time for neutrophils from myeloblasts to release of mature neutrophils is $\sim 14$ days, $\sim 60 \%$ of all nucleated cells in the bone marrow are neutrophils or their precursors. ${ }^{2}$ Neutrophils are packed with cytoplasmic granules, which contain a variety of proteins, many of which have antimicrobial properties. The human neutrophil peptides (HNP)-1 to 4 are quantitatively the most important of these, constituting at least $30 \%$ of the protein content of azurophil granules in which they are stored. ${ }^{3}$ They are small, cationic and cysteine-rich peptides with activity against a broad range of microbes. ${ }^{4}$ Their antimicrobial activities rely on several mechanisms of which membrane permeabilization is central. ${ }^{5}$ In addition, HNPs have been reported to display toxininactivating and immunomodulatory effects. ${ }^{6,7}$

Expression of the myeloid defensins HNP-1 to 4 is initiated at the late promyelocytic stage of neutrophil differentiation. Transcription of myeloid defensins relies largely on the transcription factor CCAAT/enhancer-binding protein $\varepsilon$, a key regulator of the transition from promyelocytes to myelocytes and an essential transcription factor for granule proteins localized to specific granules such as lactoferrin and hCAP-18. Most azurophil granule proteins such as myeloperoxidase (MPO) and the serine proteases such as elastase, cathepsin $\mathrm{G}$ and proteinase 3 are independent of CCAAT/enhancer-binding protein $\varepsilon$ and are expressed earlier during granulopoiesis than HNPs. ${ }^{8}$ HNPs are synthesized as 94-amino-acid (aa) preprodefensins, which are cotranslationally converted into a 75 aa proHNP form by cleavage of the common 19 aa endoplasmatic reticulum signal sequence. The 45 aa propiece is common to all four defensins. In promyelocytes, this propiece is cleaved off and degraded, whereas the mature 29 to 30-aa defensins are stored in azurophil granules by a mechanism involving electrostatic interaction with the proteoglycan serglycin., ${ }^{9,10}$ However, most HNPs are synthesized by myelocytes and metamyelocytes in which the 75 aa proHNPs are not further processed. ${ }^{11}$ ProHNPs are poorly retained in the cells, and most are secreted with only negligible quantities retained in specific granules. ${ }^{12}$ Large amounts of proHNPs are thus secreted to bone marrow plasma and further on to blood, where they can be found in $\mu \mathrm{g} / \mathrm{ml}$ concentrations in healthy individuals. $^{13}$

Because of the unique biosynthetic features mentioned above, and because no other human tissues express proHNPs, ${ }^{14}$ we hypothesized that plasma concentration of proHNPs might serve as a highly specific marker of myelopoiesis (Figure 1). Specifically, we expected levels of proHNPs to increase in plasma several days before the reappearance of neutrophils in circulation in the setting of bone marrow reconstitution after bone marrow transplantation or high-dose chemotherapy.

The neutrophil precursors can be divided into the mitotic pool, which includes myeloblasts, promyelocytes and myelocytes, and the postmitotic pool encompassing metamyelocytes, band cells and polymorphonuclear neutrophils. As proHNPs are primarily secreted from myelocytes, the last precursor stage in the mitotic pool, transit time through the postmitotic pool would be a fair initial estimate of the time from start of rise of proHNPs until neutrophil appearance in circulation. Studies employing tritiated thymidine have found a postmitotic transit time of about 6.5 days. ${ }^{15}$ When recombinant granulocyte colony-stimulating factor (G-CSF) is administered, this is reduced to 2.9 days. ${ }^{16}$

A specific marker of normal granulopoiesis would be of considerable value to clinicians. It would allow for prediction of duration of neutropenia following chemotherapy, and thus assist

The Granulocyte Research Laboratory, Department of Hematology, National University Hospital, University of Copenhagen, Copenhagen, Denmark. Correspondence: Professor N Borregaard, The Granulocyte Research Laboratory, Department of Hematology, National University Hospital-9322, University of Copenhagen, Blegdamsvej 9, Copenhagen DK-2100, Denmark. 
estimation of the risk of bacterial and fungal infections in these patients while neutropenic. In addition, such marker might alleviate the need for some of the bone marrow biopsies now taken due to suspected graft failure in the setting of prolonged pancytopenia following stem cell transplantation (SCT) or due to suspicion of failure to induce remission after chemotherapy for acute leukemia. As proHNPs are not expressed before the late promyelocyte stage, they will not be expressed by most leukemic blast cells, and would therefore be a marker of normal but not malignant myelopoiesis. In addition, in contrast to the granule proteins, proHNPs are practically absent in circulating neutrophils, and the level in plasma is thus a specific marker of myelopoietic activity.

In the present study we investigated for the first time this clinical aspect of proHNP biology by measuring proHNPs in plasma of patients undergoing SCT or chemotherapy for acute leukemia and correlating the proHNP levels to circulating neutrophil counts.

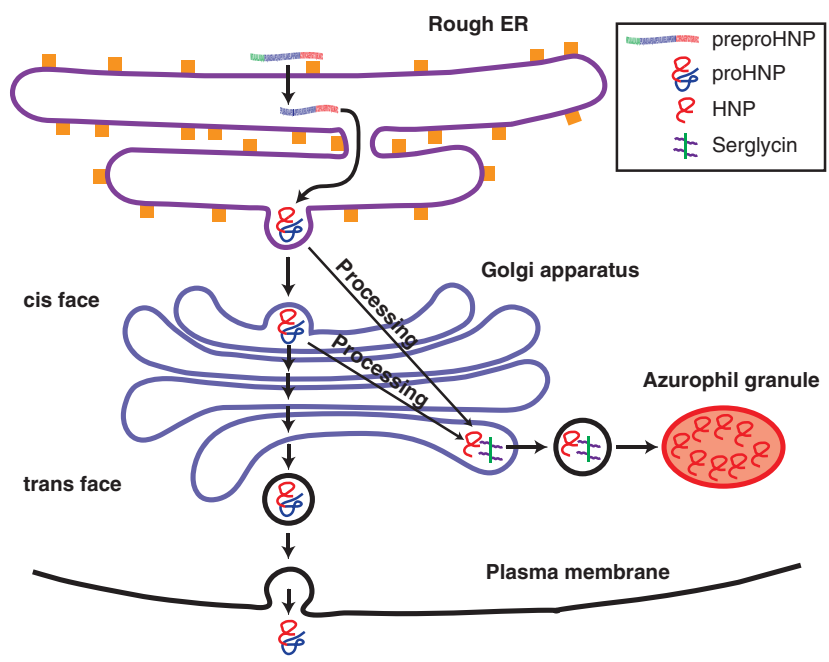

Figure 1. Biosynthetic pathway of HNPs. PreproHNPs are cleaved cotranslationally into proHNPs. In the promyelocyte, most proHNPs are processed by a yet unidentified protease into mature cationic HNPs. HNPs are able to associate with the anionic proteoglycan serglycin, which aides in translocation to azurophil granules. In myelocytes and metamyelocytes, the electrically neutral proHNPs are not processed. This hinders association with serglycin and most proHNP is secreted into the bone marrow plasma, although a minor part is retained in specific granules. HNP, human neutrophil peptides.

\section{MATERIALS AND METHODS}

\section{Ethics statement}

Human peripheral blood samples were obtained after informed consent according to the permission (H-1-2012-030) and guidelines from the local ethics committee of the Capital Region of Denmark.

\section{Patients}

Forty-one patients were included from the Department of Hematology, National University Hospital, Copenhagen, Denmark.

Blood samples were collected in $3 \mathrm{ml}$ EDTA tubes (Vacuette, Greiner Bio-One, Kremsmünster, Austria). After centrifugation at $1753 \mathrm{~g}$ for $10 \mathrm{~min}$, plasma was taken from the tubes and frozen at $-80^{\circ} \mathrm{C}$.

Plasma levels of proHNPs, MPO, lysozyme and neutrophil gelatinaseassociated lipocalin (NGAL) were determined by enzyme-linked immunosorbent assay (ELISA) in three patient cohorts: patients receiving allogeneic stem cells after myeloablative conditioning (xSCT), patients undergoing autologous SCT (aSCT) and patients undergoing induction or consolidation chemotherapy for acute leukemia. Furthermore, plasma levels of proHNPs, MPO and lysozyme were determined in 19 patients with acute myeloid leukemia (AML) before chemotherapy and in 39 healthy controls.

In the XSCT and aSCT patients, blood sampling was initiated at day 0 , that is, the day of stem cell infusion, and continued until patients were discharged. For patients receiving induction or consolidation chemotherapy, blood sampling was initiated during chemotherapy and continued until neutrophil recovery or discharge of the patient.

A main objective in the analysis of data was to determine the time by which the rise in plasma levels of each of the four neutrophil proteins preceded neutrophil reappearance. Neutrophil counts were by automated differential counts using the Sysmex XE-2100 (Sysmex Corporation, Kobe, Japan). This was done when total leukocytes were $>0.5 \times 10^{9}$ per I blood.

PPI (ProHNP-PMN-Interval) will be used to denote the timespan between reappearance of proHNPs in plasma and reappearance of neutrophils in circulation. Myeloperoxidase PMN interval, lysozyme PMN interval, and neutrophil gelatinase-associated lipocalin PMN interval will be used for MPO, lysozyme and NGAL, respectively, but as these peptides do not disappear from circulation as proHNPs do, they will denote the timespan from the preincrease trough value to reappearance of neutrophils in circulation.

\section{Antibodies}

The following antibodies were used: rabbit anti-proHNP, ${ }^{12}$ rabbit anti-MPO (A0398; Dako, Glostrup, Denmark), rabbit anti-lysozyme (A0099; Dako) and polyclonal rabbit anti-NGAL, as well as biotinylated monoclonal mouse anti-NGAL for the NGAL-ELISA. ${ }^{17}$ All antibodies were biotinylated in-house as described. ${ }^{18}$

\section{ELISA}

ELISAs were performed as previously described. ${ }^{19}$ Specifications on antibodies and standards used in the various ELISAs are described elsewhere: ProHNP, ${ }^{13} \mathrm{MPO}^{20}$ lysozyme ${ }^{21}$ and NGAL. ${ }^{17}$

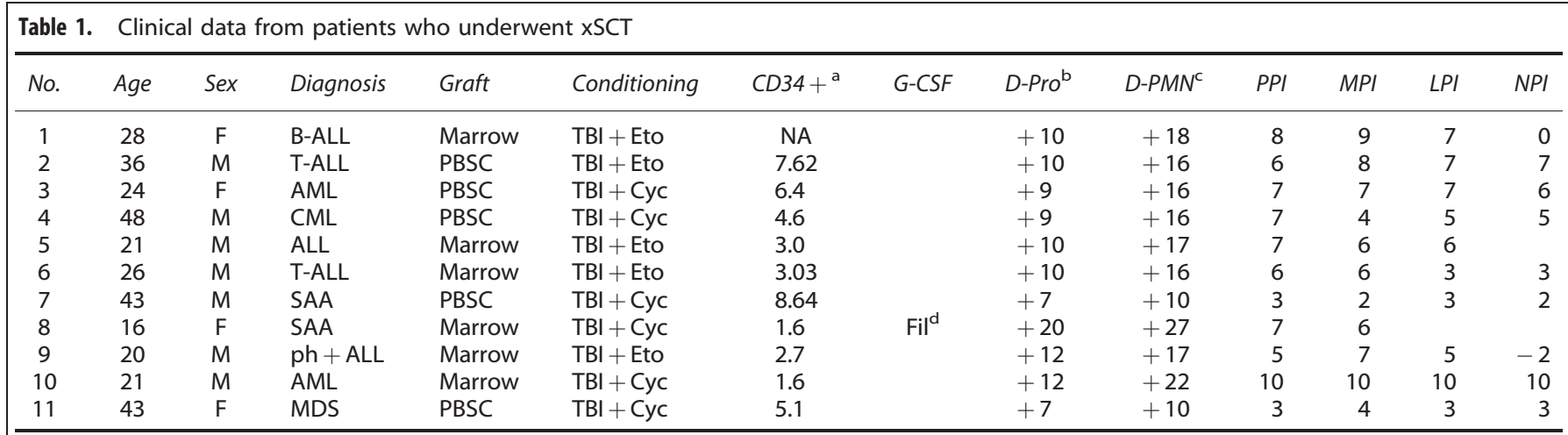

Abbreviations: ALL, acute lymphoid leukemia; AML, acute myeloid leukemia; Cyc, cyclophosphamide; $C M L$, chronic myeloid leukemia; Eto, etoposid; $F$, female; G-CSF, granulocyte colony-stimulating factor; LPI, lysozyme PMN interval; M, male; MDS, myelodysplastic syndrome; MPI, myeloperoxidase PMN interval; NPI, neutrophil gelatinase-associated lipocalin PMN interval; PBSCs, peripheral blood stem cells; PMN, polymorphonuclear neutrophil; PPI, ProHNP-PMN-Interval; SAA, severe aplastic anemia; TBI, total body irradiation; xSCT, allogenic stem cell transplantation. ${ }^{\mathrm{a}} \mathrm{Graft} \mathrm{CD} 34+$ cell count, $10^{6}$ cells $/ \mathrm{kg}$ bodyweight. ${ }^{\mathrm{b}}$ Day of proHNP reappearance. ${ }^{\mathrm{C}} \mathrm{Day}$ of PMN reappearance. ${ }^{\mathrm{d}}$ Filgrastim $0.3 \mathrm{mg}$ from day +24 to day +34 . 


\section{Statistical analyses}

Statistical calculations were performed with Graphpad 5.0 (Graphpad Software, La Jolla, CA, USA). Differences in levels of plasma proteins between patients with AML and healthy controls were analyzed by MannWhitney $U$-test $(P<0.05$ was regarded as significant).

\section{RESULTS}

\section{Allogeneic transplantations}

Table 1 provides clinical data on the 11 patients in this group. Figure $2 a$ shows data from patient 3 . This patient's pattern of proHNP reappearance is typical of the group.

Some of the xSCT patients were profoundly neutropenic already at day 0 and the rest had neutrophil counts that rapidly declined, such that neutrophils had disappeared from circulation of all patients by day +5 , in line with the expected effects of myeloablative conditioning. Plasma concentration of proHNPs followed a course that mimicked this, such that proHNPs had disappeared from plasma no later than day +5 in all patients. In two patients, we observed a small rise in plasma proHNPs on day +1 , indicating that the allografts contained some proHNPs.

There was variation (day +7 to day +20 ) in the time until proHNP reappearance in plasma, most likely reflecting the known variation in engraftment time, but in all 11 patients, proHNPs reappeared in plasma before reappearance of neutrophils in circulation. The average PPI was 6.3 days (range 3 to 10, s.d. 2.1). In patients 7 and 11, engraftment was swift and proHNP reappearance occurred only 3 days before neutrophil reappearance,
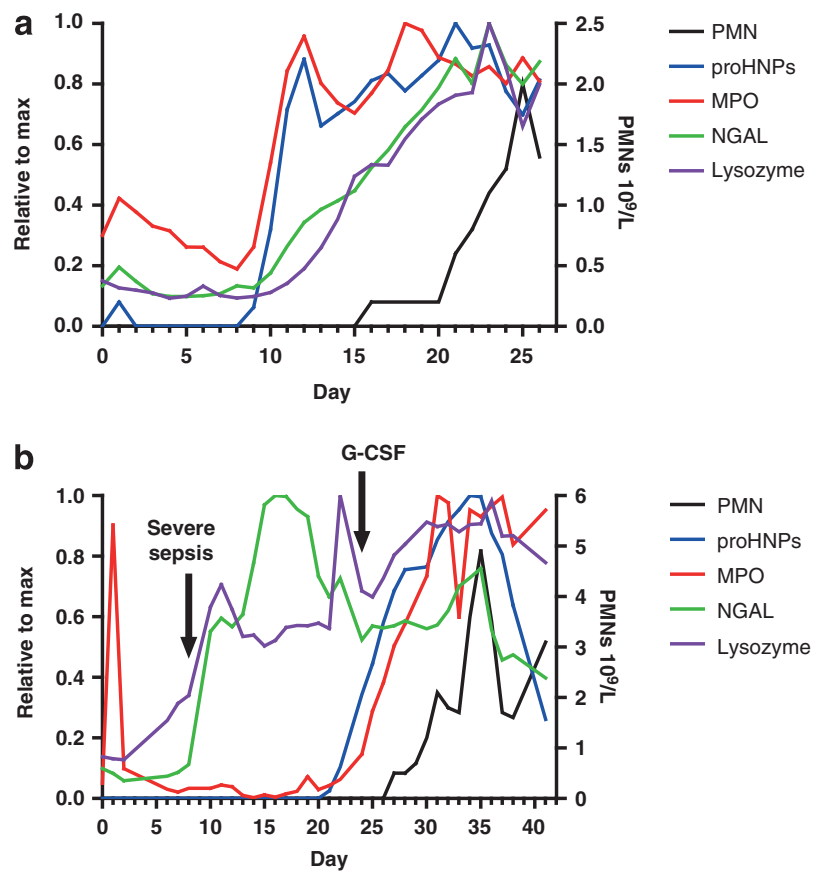

Figure 2. ProHNPs, MPO, lysozyme, NGAL and PMNs in peripheral blood of two patients who underwent xSCT. Plasma levels of proHNPs, MPO, NGAL and lysozyme were measured by ELISA and related to neutrophil count in circulation. Day 0 marks the day of xSCT. (a) Values from patient 3 are depicted here, showing a pattern typical for this group. It is seen that only proHNPs disappear completely from circulation. (b) Values from patient 8 who developed severe sepsis at day 8 . Note the rising plasma levels of lysozyme and NGAL in conjunction with infection, whereas proHNPs and MPO followed neutrophil engraftment. HNP, human neutrophil peptides; MPO, myeloperoxidase; NGAL, neutrophil gelatinase-associated lipocalin; PMNs, polymorphonuclear neutrophils; SCT, stem cell transplantations. indicating that in patients with a successful, early engraftment, granulopoiesis seems to be accelerated.

MPO and lysozyme were both reliable predictors of neutrophil reappearance in these patients, with a mean myeloperoxidase PMN interval of 6.3 days (s.d. 2.33) and a mean lysozyme PMN interval of 5.6 days (s.d. 2.27). Levels of NGAL generally increased later, as witnessed by a mean neutrophil gelatinase-associated lipocalin PMN interval of 3.77 (s.d. 3.66). In patient 5 there was no clearly discernible rise of NGAL, and in patient 9 NGAL started to rise only after neutrophils had already appeared in plasma. ProHNP was the only granulocyte protein that consistently disappeared from plasma.

Patient 8 developed septic shock with blood cultures positive for Staphylococcus haemolyticus and acute kidney injury with a peak creatinine of $542 \mu \mathrm{mol} / \mathrm{l}$, and this coincided with a marked rise in levels of NGAL and lysozyme (Figure 2b). This most likely reflected non-myeloid expression, as NGAL is an established marker of acute kidney injury, ${ }^{22,23}$ and lysozyme is induced in a number of tissues in response to inflammation. ${ }^{24}$ Also note that proHNPs and MPO remained stable during the systemic infection, their eventual rise reflecting myelopoiesis.

The maximum concentrations of proHNPs reached within the sampling period ranged from 4.17 to $9.58 \mu \mathrm{g} / \mathrm{ml}$ among the 10 patients who did not receive G-CSF. Patient 8 received G-CSF from day +24 , and in this patient the concentration of proHNPs peaked at $32.39 \mu \mathrm{g} / \mathrm{ml} 10$ days after initiation of G-CSF. The reason for initiating therapy with G-CSF was that the total leukocyte count was still below $0.1 \times 10^{9} /$ by day +24 . ProHNPs had however started to rise at day +20 , heralding onset of myelopoietic activity. A bone marrow biopsy was planned for day +30 to evaluate marrow cellularity, but was cancelled when neutrophils started to rise by day +27 .

\section{Autologous transplantations}

Table 2 shows some key clinical characteristics of these 16 patients. Figure $3 a$ shows a representative pattern of proHNPs and neutrophils in plasma from one of these patients. In all but one patient in this group, the conditioning chemotherapy led to disappearance of both neutrophils and proHNPs from circulation. Thus, with the exception of patient 17, in whom proHNPs persisted in plasma (see below), neutrophils and proHNPs had disappeared from plasma no later than day +5 and +4 , respectively.

All these patients received G-CSF $(5 \mu \mathrm{g} / \mathrm{kg})$ from day +5 as part of their supportive treatment, and this clearly affected the kinetics of increase in proHNPs and neutrophil reappearance, shortening the time between the two, compared with the corresponding kinetics in the allogeneic transplantation setting.

The average PPI in this group was 2.8 days (range 1 to 4 , s.d. 0.8 ). The pattern of proHNP rise was more uniform in this group, and the maximal plasma concentrations of proHNPs reached were higher than in the allogeneic group (average $26.8 \mu \mathrm{g} / \mathrm{ml}$, range 12.1 to $44.3 \mu \mathrm{g} / \mathrm{ml}$ ).

As seen in Figure 3a, there was a tendency for plasma levels of proHNPs to reach a peak, and then decrease and settle on a somewhat lower level. This initial peak occurs while the patients are still receiving G-CSF, and the subsequent decline coincides with cessation of G-CSF treatment. G-CSF is routinely withdrawn when neutrophil counts reach $0.5 \times 10^{9} /$.

In patient 17, proHNPs remained in plasma throughout the sampling period (Figure $3 \mathrm{~b}$ ). This myeloma patient had-by farthe highest level of proHNPs at day 0 observed in our study. Her kidney function was normal and the conditioning regimen did not differ from that of the five other myeloma patients. As expected, she also entered a profound neutropenia.

Induction chemotherapy

Table 3 provides some clinical characteristics of the 14 patients in this group. 
Table 2. Clinical data from patients who underwent aSCT

\begin{tabular}{|c|c|c|c|c|c|c|c|c|}
\hline No. & Age & Sex & Diagnosis & Conditioning & $C D 34+{ }^{a}$ & D-Pro ${ }^{\mathrm{b}}$ & $D-P M N^{c}$ & PPI \\
\hline 12 & 58 & $\mathrm{~F}$ & T-cell lymphoma & BEAM & 5.1 & +6 & +9 & 3 \\
\hline 13 & 62 & M & NKT-cell lymphoma & BEAM & 2.9 & +5 & +9 & 4 \\
\hline 14 & 65 & $\mathrm{~F}$ & Richter transformation of CLL & BEAM & 3.6 & +8 & +10 & 2 \\
\hline 15 & 62 & M & MM & HD Mel & 8.1 & +9 & +12 & 3 \\
\hline 16 & 66 & $\mathrm{~F}$ & DLBCL & BEAM & 2.7 & +7 & +10 & 3 \\
\hline 17 & 64 & $\mathrm{~F}$ & MM & HD Mel & 6.8 & +7 & +11 & 4 \\
\hline 18 & 51 & M & DLBCL & BEAM & 8.1 & +7 & +10 & 3 \\
\hline 19 & 67 & $\mathrm{~F}$ & $\mathrm{FL}$ & BEAM & 3.4 & +7 & +10 & 3 \\
\hline 20 & 35 & M & T-cell lymphoma & BEAM & 10.9 & +8 & +10 & 2 \\
\hline 21 & 65 & $\mathrm{~F}$ & MM & HD Mel & 2.3 & +7 & +9 & 2 \\
\hline 22 & 57 & $\mathrm{~F}$ & FL & BEAM & 2.4 & +7 & +10 & 3 \\
\hline 23 & 66 & M & T-ALL & $\mathrm{Bu}+\mathrm{Cyc}$ & 3.4 & +8 & +11 & 3 \\
\hline 24 & 58 & $M$ & CML and DLBCL & R-BCNÚ-Tep & 5.9 & +6 & +9 & 3 \\
\hline 25 & 51 & $\mathrm{~F}$ & MM & HD Mel & 13.4 & +8 & +11 & 3 \\
\hline 26 & 67 & $\mathrm{~F}$ & MM & HD Mel & 2.6 & +9 & +10 & 1 \\
\hline 27 & 65 & $\mathrm{~F}$ & MM & HD Mel & 6.3 & +7 & +10 & 3 \\
\hline
\end{tabular}

Abbreviations: ALL, acute lymphoid leukemia; aSCT, autologous stem cell transplantation; BEAM, carmustin, etoposid, cytarabin, melphalan; Bu + cyc, busulfan + cyclophosphamide; CLL, chronic lymphocytic leukemia; $C M L$, chronic myeloid leukemia; $D L B C L$, diffuse large $B$-cell lymphoma; $F$, female; $F L$, follicular lymphoma; HD Mel, high-dose melphalan; M, male; MM, multiple myeloma; NKT, natural killer T-cells; PMN, polymorphonuclear neutrophil; PPI, ProHNP-PMN-Interval; R-BCNU-Tep, rituximab, carmustine, thiotepa. ${ }^{\mathrm{a}} \mathrm{Graft} \mathrm{CD} 34$ + cell count, $10^{6} \mathrm{cells} / \mathrm{kg}$ bodyweight. ${ }^{\mathrm{b}}$ Day of proHNP reappearance (in patient 17 it signifies start of rise rather than reappearance). ${ }^{\circ}$ Day of PMN reappearance.
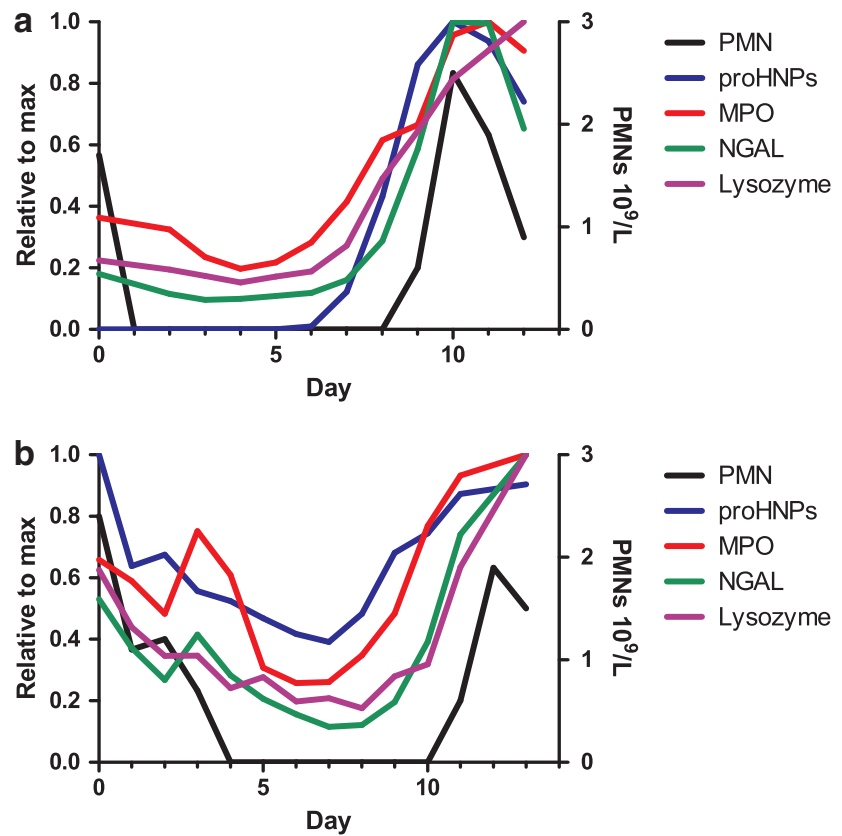

Figure 3. ProHNPs, MPO, lysozyme, NGAL and PMNs in peripheral blood of two patients who underwent aSCT. (a) Figure based on data from patient 12, displaying a pattern representative of the group. All four assayed proteins increase approximately simultaneously, and PPI is shorter than that in the XSCT group. Again, only proHNPs disappear from circulation following myeloablative conditioning. (b) The only example in our study of a patient (17) in whom proHNPs remained in plasma following chemotherapy. HNP, human neutrophil peptides; MPO, myeloperoxidase; NGAL, neutrophil gelatinase-associated lipocalin; PMNs, polymorphonuclear neutrophils; PPI, ProHNP-PMN-Interval; SCT, stem cell transplantations.

Although the treatment given to this group of patients was not myeloablative, it did suppress myelopoiesis sufficiently to make proHNPs disappear from circulation. In general, the patients receive chemotherapy while admitted to the ward, but receive transfusions on an out-patient basis every other day during the period of profound cytopenia. Blood was thus sampled for determination of circulating blood cell levels only every other day. The PPI was therefore more difficult to determine accurately in this group of patients. In spite of this sub-optimal sampling of blood for proHNP assay, it is clear that proHNPs in plasma precede neutrophils in blood and that the PPI seems to vary between 2 and 7 days.

Patient 35 had AML secondary to myelodysplastic syndrome. In this patient, chemotherapy likely resulted in reversion to the myelodysplastic syndrome stage, and there was an extraordinarily long gap between the rise of MPO and lysozyme, and the reemergence in proHNPs and NGAL, most likely reflecting that MPO and lysozyme are both expressed by myeloblasts and promyelocytes, and proHNPs and NGAL representing more mature cells which appear later ${ }^{25}$ and to a lesser extent in myelodysplastic syndrome (Figure 4a).

In patient 38, chemotherapy failed to induce bone marrow remission as ascertained by bone marrow examination performed 38 days after initiation of chemotherapy. This patient suffered from acute monocytic leukemia in which blasts are known to be high in lysozyme content, whereas barely expressing MPO. ${ }^{26}$ In accordance with this, we found an early increase in lysozyme, but this time with a small decrease in MPO, and again not followed by reappearance of proHNP or neutrophils (Figure $4 \mathrm{~b}$ ). Thus, in this patient, the rise of lysozyme most likely represents reappearing malignant myelopoiesis, whereas the late and minor rise of proHNPs reflects normal myelopoiesis. ProHNPs only rose to 0.98 and $2.1 \mu \mathrm{g} / \mathrm{ml}$ in 35 and 38 , respectively, indicating that normal myelopoiesis was compromised in both patients.

\section{Pre-induction AML}

ProHNPs are not produced until the late promyelocyte and myelocytic stages of neutrophil differentiation, that is, in myeloid cells that are more mature than cells from the leukemic clones of AML. To assert that proHNPs were not significantly secreted by malignant cells in AML, levels of proHNPs, MPO and lysozyme were determined by ELISA in pretreatment plasma samples from 19 AML patients (samples taken before initiation of chemotherapy, that is, at a time of considerable malignant myelopoiesis) and from 39 healthy controls. Concentration of proHNPs in plasma of 
Table 3. Clinical data from patients who underwent chemotherapy for acute leukemias

\begin{tabular}{|c|c|c|c|c|c|c|c|}
\hline No. & Age & Sex & Diagnosis & Treatment & G-CSF & $P P I$ & Peak proHNPs $(\mu \mathrm{g} / \mathrm{ml})$ \\
\hline 28 & 45 & $\mathrm{~F}$ & AML & HD Cytosar & & $3-5$ & 7.7 \\
\hline 29 & 45 & $\mathrm{~F}$ & AML & HD Cytosar & & $4-6$ & 4.5 \\
\hline 30 & 57 & M & ALL & Hyper-CVAD & Filgrastim $0.96 \mathrm{mg}$ daily & 5 & 33.7 \\
\hline 31 & 55 & $\mathrm{~F}$ & AML & HD Cytosar & & - & 16.2 \\
\hline 32 & 66 & $\mathrm{~F}$ & AML & $\mathrm{Da} 3+10$ & & $3-5$ & 20.2 \\
\hline 33 & 66 & $\mathrm{~F}$ & AML & Da $3+8$ & & $2-5$ & 20.4 \\
\hline 34 & 67 & M & AML & $\mathrm{Da} 3+10$ & & 4 & 28.8 \\
\hline 35 & 51 & M & AML & Da $3+10$ & & - & 0.98 \\
\hline 36 & 44 & $\mathrm{~F}$ & AML & FLAG-IDA & Lenograstim $0.263 \mathrm{mg}$ daily & $3-5$ & 19.2 \\
\hline 37 & 51 & M & AML & $\mathrm{Da} 3+10$ & & $5-7$ & 18.1 \\
\hline 38 & 70 & M & AML & FLAG-Nov & Filgrastim $0.30 \mathrm{mg}$ daily & - & 2.1 \\
\hline 39 & 23 & M & AML & $\mathrm{Da} 3+10$ & Pegfilgrastim $6 \mathrm{mg} \times 1$ & $5-7$ & 36.3 \\
\hline 40 & 70 & M & AML & FLAG & Filgrastim $0.30 \mathrm{mg}$ daily & 5 & 18.6 \\
\hline 41 & 65 & M & AML & $\mathrm{Da} 3+10$ & & 7 & 24.1 \\
\hline
\end{tabular}

Abbreviations: ALL, acute lymphoid leukemia; AML, acute myeloid leukemia; Da 3+8, daunorubicin, cytarabine; Da $3+10$, daunorubicin, cytarabine; F, female; FLAG-Ida, fludarabine, cytarabine, lenograstim, idarubicin; FLAG-Nov, fludarabine, cytarabine, filgrastim, mitoxantron; G-CSF, granulocyte colony-stimulating factor; HD Cytosar, high-dose cytarabine; HNP, human neutrophil peptides; Hyper-CVAD, cyclophosphamid, vincristin, cytarabine, doxorubicin, intrathecal methotrexate; M, male; PPI, ProHNP-PMN-Interval.
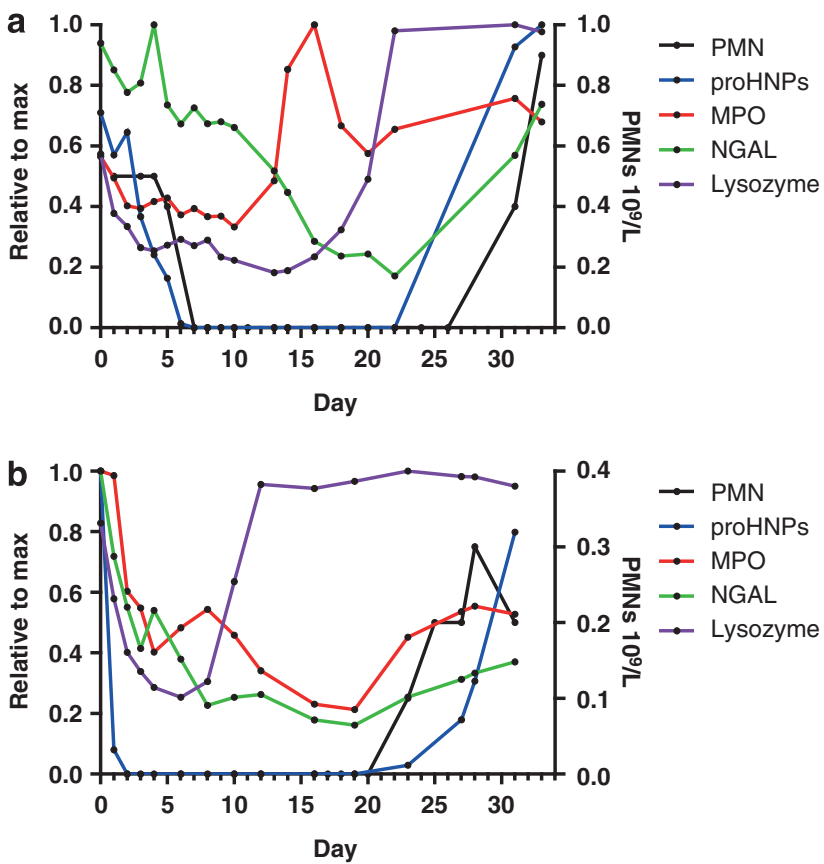

Figure 4. ProHNPs, MPO, lysozyme, NGAL and PMNs in peripheral blood of two patients who underwent induction chemotherapy for AML. (a) Patient 35 in whom MPO and lysozyme increased early, possibly reflecting this patient's underlying myelodysplasia. The eventual rise of proHNPs was linked to reappearance of neutrophils in circulation. (b) Patient 38 in whom lysozyme increased early, most likely signifying reappearance of malignant myelopoiesis. AML, acute myeloid leukemia; HNP, human neutrophil peptides; MPO, myeloperoxidase; NGAL, neutrophil gelatinase-associated lipocalin; PMNs, polymorphonuclear neutrophils.

AML patients who had not received chemotherapy was significantly lower than that of healthy controls $(P<0.0001$; Figure 5). Levels of MPO and lysozyme did not differ significantly between patients and controls (MPO: $P=0.55$; lysozyme: $P=0.13$; Figure 5 ).

\section{DISCUSSION}

In previous studies, concentrations of MPO, lactoferrin and lysozyme were all found to increase in blood before reappearance of neutrophils, following allogeneic bone marrow transplantation. $^{27,28}$ For MPO and lysozyme, we confirm these findings. These proteins rose in plasma on average 6.3 and 5.6 days before reappearance of neutrophils, approximately the same as the PPI in this group of patients. NGAL generally showed less promise as a predictor of neutrophil reappearance, rising no earlier than neutrophils in patients 1 and 9, and showed no clearly discernible rise in patient 5. NGAL is expressed in several nonmyeloid tissues ${ }^{29}$ including damaged kidney cells and is thus considered an excellent early marker of acute kidney injury. ${ }^{22,23}$ Lysozyme is expressed in a range of tissues ${ }^{30}$ and is induced in resident macrophages on inflammation. ${ }^{31}$ Plasma levels of NGAL and lysozyme are thus not specific markers of myelopoiesis. This was witnessed in patient 8 (Figure 2) who developed septic shock with renal failure, and in whom an increase in lysozyme and NGAL was seen, which was not followed by neutrophil reappearance, thus obviously rendering these proteins less reliable as markers of myelopoiesis.

In addition to these findings, the present study displayed in particular one important feature of proHNPs that distinguished them from MPO, lysozyme and NGAL, namely, the fact that they disappeared from circulation in 40 out of 41 patients. This increases the clinical usefulness of proHNPs, because their presence in plasma is a specific marker of normal myelopoietic activity. Although MPO is exclusively expressed by neutrophil and monocyte precursors, ${ }^{32}$ MPO is by no means a marker of normal myelopoiesis, as MPO is expressed in most AML and is used as a diagnostic to distinguish AML from ALL. In contrast, proHNPs are exclusively expressed by neutrophil precursors and are not expressed by AML blasts (Figure 5). The one patient with persistent proHNPs in plasma also had-by far-the highest initial concentration. As with the other aSCT patients, she responded to the conditioning with profound neutropenia, making it unlikely that she had any residual myelopoietic activity. However, the use of G-CSF shortened the timeframe with no myelopoiesis, which may have been inadequate for the total clearance of plasma proHNPs in this patient.

G-CSF is known to accelerate both proliferation and maturation of neutrophil precursors. ${ }^{33}$ If plasma concentration of proHNPs does reflect myelopoietic activity, we would expect G-CSF administration to increase the release of proHNPs from bone marrow (proliferation) and to shorten PPI (maturation). This was indeed found in the aSCT patients who all received G-CSF from day +5 .

In conclusion, our study demonstrates that proHNPs are markers of normal, but not malignant, myelopoiesis, and that rise 

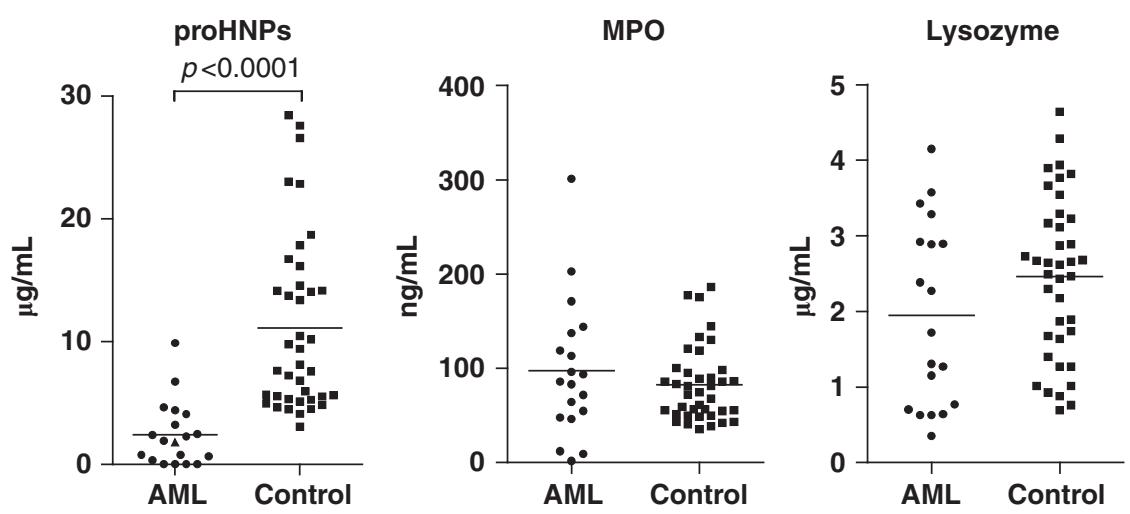

Figure 5. Plasma levels of proHNPs, MPO and lysozyme in patients with AML. Peripheral blood samples were taken from 19 patients with AML at diagnosis before chemotherapy and 39 healthy controls. Levels of proHNPs (left), MPO (middle) and lysozyme (right) were measured by ELISA. ProHNPs were significantly lower in AML patients compared with healthy controls $(P<0.0001)$. Levels of MPO and lysozyme did not differ significantly between patients and controls (MPO: $P=0.55$ and lysozyme: $P=0.13$ ). Differences were analyzed by Mann-Whitney $U$-test AML, acute myeloid leukemia; HNP, human neutrophil peptides; MPO, myeloperoxidase; NGAL, neutrophil gelatinase-associated lipocalin.

of plasma proHNPs reflects that normal hematopoiesis has been established, even in the absence of detectable circulating neutrophils, as demonstrated here in the setting of xSCT and aSCT, and in the treatment of acute leukemia. It is possible that measurement of proHNPs can also be of value in the workup of patients with neutropenia, with very low or very high values yielding information about failing granulopoiesis (agranulocytosis) or increased compensatory granulopoiesis (immune-mediated neutrophil destruction), respectively. Further studies are needed to clarify this, and will hopefully expand the potential clinical value of measurement of proHNPs in plasma.

\section{CONFLICT OF INTEREST}

The authors declare no conflict of interest.

\section{ACKNOWLEDGEMENTS}

The expert technical assistance of Charlotte Horn is greatly acknowledged. This work was supported by grants from The Danish Council for Independent Research (NB).

\section{AUTHOR CONTRIBUTIONS}

FE performed experiments; FE and AG included patients and obtained samples; FE, AG and NB designed the research and analyzed results; and PK, JS and HS participated in the design of the study and inclusion of patients. All authors contributed to the writing of the paper.

\section{REFERENCES}

1 Mary JY. Normal human granulopoiesis revisited. II. Bone marrow data. Biomed Pharmacother 1985; 39: 66-77.

2 Bainton DF, Ullyot JL, Farquhar MG. The development of neutrophilic polymorphonuclear leukocytes in human bone marrow. J Exp Med 1971; 134: 907-934.

3 Rice WG, Ganz T, Kinkade JM, Selsted ME, Lehrer Rl, Parmley RT. Defensin-rich dense granules of human neutrophils. Blood 1987; 70: 757-765.

4 Ganz T, Selsted ME, Szklarek D, Harwig SS, Daher K, Bainton DF et al. Defensins. Natural peptide antibiotics of human neutrophils. J Clin Invest 1985; 76: 1427-1435.

5 Zhang Y, Lu W, Hong M. The membrane-bound structure and topology of a human $\alpha$-defensin indicate a dimer pore mechanism for membrane disruption. Biochemistry 2010; 49: 9770-9782.

6 Biragyn A. Defensins--non-antibiotic use for vaccine development. Curr Protein Pept Sci 2005; 6: 53-60.

7 Lehrer RI. Multispecific myeloid defensins. Curr Opin Hematol 2007; 14: 16-21.

8 Khanna-Gupta A, Zibello T, Sun H, Gaines P, Berliner N. Chromatin immunoprecipitation (ChIP) studies indicate a role for CCAAT enhancer binding proteins alpha and epsilon (C/EBP alpha and C/EBP epsilon ) and CDP/cut in myeloid maturation-induced lactoferrin gene expression. Blood 2003; 101: 3460-3468.
9 Valore EV, Ganz T. Posttranslational processing of defensins in immature human myeloid cells. Blood 1992; 79: 1538-1544.

10 Glenthøj A, Cowland JB, Heegaard NH, Larsen MT, Borregaard N. Serglycin participates in retention of $\alpha$-defensin in granules during myelopoiesis. Blood 2011; 118: 4440-4448.

11 Arnljots K, Sørensen O, Lollike K, Borregaard N. Timing, targeting and sorting of azurophil granule proteins in human myeloid cells. Leukemia 1998; 12: 1789-1795

12 Faurschou M, Kamp S, Cowland JB, Udby L, Johnsen AH, Calafat J et al. Prodefensins are matrix proteins of specific granules in human neutrophils. $J$ Leukoc Biol 2005; 78: 785-793.

13 Glenthøj A, Glenthøj AJ, Borregaard N. ProHNPs are the principal $\alpha$-defensins of human plasma. Eur J Clin Invest 2013; 43(8): 836-843.

14 Date $\mathrm{Y}$, Nakazato M, Shiomi K, Toshimori H, Kangawa K, Matsuo $\mathrm{H}$ et al. Localization of human neutrophil peptide (HNP) and its messenger RNA in neutrophil series. Ann Hematol 1994; 69: 73-77.

15 Dancey JT, Deubelbeiss KA, Harker LA, Finch CA. Neutrophil kinetics in man. J Clin Invest 1976; 58: 705-715.

16 Price TH, Chatta GS, Dale DC. Effect of recombinant granulocyte colonystimulating factor on neutrophil kinetics in normal young and elderly humans. Blood 1996; 88: 335-340.

17 Kjeldsen L, Koch C, Arnljots K, Borregaard N. Characterization of two ELISAs for NGAL, a newly described lipocalin in human neutrophils. $J$ Immunol Methods 1996; 198: 155-164.

18 Bayer EA, Wilchek M. Protein biotinylation. Methods Enzymol 1990; 184: 138-160.

19 Rørvig S, Honore C, Larsson L-I, Ohlsson S, Pedersen CC, Jacobsen LC et al. Ficolin-1 is present in a highly mobilizable subset of human neutrophil granules and associates with the cell surface after stimulation with fMLP. J Leukoc Biol 2009; 86: 1439-1449.

20 Borregaard N, Kjeldsen L, Sengeløv H, Diamond MS, Springer TA, Anderson HC et al. Changes in subcellular localization and surface expression of L-selectin, alkaline phosphatase, and Mac-1 in human neutrophils during stimulation with inflammatory mediators. J Leukoc Biol 1994; 56: 80-87.

21 Lollike K, Kjeldsen L, Sengeløv H, Borregaard N. Purification of lysozyme from human neutrophils, and development of an ELISA for quantification in cells and plasma. Leukemia 1995; 9: 206-209.

22 Mishra J, Dent C, Tarabishi R, Mitsnefes MM, Ma Q, Kelly C et al. Neutrophil gelatinase-associated lipocalin (NGAL) as a biomarker for acute renal injury after cardiac surgery. Lancet 2005; 365: 1231-1238.

23 Mishra J, Ma Q, Kelly C, Mitsnefes M, Mori K, Barasch J et al. Kidney NGAL is a novel early marker of acute injury following transplantation. Pediatr Nephrol 2006; 21: 856-863.

24 Porstmann B, Jung K, Schmechta H, Evers U, Pergande M, Porstmann T et al. Measurement of lysozyme in human body fluids: comparison of various enzyme immunoassay techniques and their diagnostic application. Clin Biochem 1989; 22: 349-355.

25 Theilgaard-Mönch K, Jacobsen LC, Borup R, Rasmussen T, Bjerregaard MD, Nielsen FC et al. The transcriptional program of terminal granulocytic differentiation. Blood 2005; 105: 1785-1796. 
26 Resnitzky P, Shaft D. Distinct lysozyme content in different subtypes of acute myeloid leukaemic cells: an ultrastructural immunogold study. $\mathrm{Br} J$ Haematol 1994; 88: 357-363.

27 Oberg G, Simonsson B, Smedmyr B, Tötterman TH, Venge P. Myeloid regeneration after bone-marrow transplantation monitored by serum measurements of myeloperoxidase, lysozyme and lactoferrin. Eur J Haematol 1987; 38: 356-362.

28 Lollike K, Kjeldsen L, Sengeløv H, Borregaard N. Lysozyme in human neutrophils and plasma. A parameter of myelopoietic activity. Leukemia 1995; 9: 159-164.

29 Friedl A, Stoesz SP, Buckley P, Gould MN. Neutrophil gelatinase-associated lipocalin in normal and neoplastic human tissues. Cell type-specific pattern of expression. Histochem J 1999; 31: 433-441.

30 Su Al, Wiltshire T, Batalov S, Lapp H, Ching KA, Block D et al. A gene atlas of the mouse and human protein-encoding transcriptomes. Proc Natl Acad Sci USA 2004; 101: 6062-6067.
31 Keshav S, Chung P, Milon G, Gordon S. Lysozyme is an inducible marker of macrophage activation in murine tissues as demonstrated by in situ hybridization. J Exp Med 1991; 174: 1049-1058.

32 Hansson M, Olsson I, Nauseef WM. Biosynthesis, processing, and sorting of human myeloperoxidase. Arch Biochem Biophys 2006; 445: 214-224.

33 Lord BI, Molineux G, Pojda Z, Souza LM, Mermod JJ, Dexter TM. Myeloid cell kinetics in mice treated with recombinant interleukin-3, granulocyte colonystimulating factor (CSF), or granulocyte-macrophage CSF in vivo. Blood 1991; 77: 2154-2159.

(1) $=$ This work is licensed under a Creative Commons AttributionBY NC ND NonCommercial-NoDerivs 3.0 Unported License. To view a copy of this license, visit http://creativecommons.org/licenses/by-nc-nd/3.0/ 